\title{
Incidence of Post Cross Clamp Ventricular Fibrillation in Isolated Coronary Artery Bypass Surgery Using del Nido Cardioplegia and Conventional Blood Cardioplegia
}

\author{
Biju Kambil Thyagarajan¹, Anandakuttan Sreenivasan², Ravikrishnan Jayakumar ${ }^{2}$, Ratish Radhakrishnan ${ }^{4}$ \\ 1,2,3,4 Department of Cardiovascular and Thoracic Surgery, \\ Government T D Medical College, Alappuzha, Kerala, India.
}

\section{ABSTRACT}

\section{BACKGROUND}

The cardiac surgical procedures and surgical outcomes witnessed a dramatic improvement with the introduction of cardiopulmonary bypass and cardioplegia techniques. Ventricular fibrillation immediately after removal of aortic cross clamp is an energy consuming process leading to myocardial injury in an already energy depleted heart. Electrical cardioversion, which itself causes myocardial injury, is required to regain normal rhythm. Prevention of ventricular fibrillation is important in preventing myocardial injury. We retrospectively analysed the incidence of post cross clamp ventricular fibrillation requiring electrical defibrillation in isolated coronary artery bypass surgery using del Nido cardioplegia and conventional blood cardioplegia.

\section{METHODS}

We retrospectively collected the data of 200 patients who underwent isolated coronary artery bypass graft surgery under cardiopulmonary bypass with cardioplegic arrest with 100 pts in del Nido cardioplegia (DNCP) group and 100 pts in conventional blood cardioplegia (CBCP) group and analysed the incidence of post cross clamp ventricular fibrillation requiring electrical defibrillation.

\section{RESULTS}

The pre-operative patient parameters, preoperative cardiac function, and number of vessels involved, the number of grafts and co-morbidities showed no statistically significant difference in both groups. Four patients in the del Nido cardioplegia group and 24 patients in the conventional blood cardioplegia group developed post aortic declamping ventricular fibrillation requiring electrical defibrillation with a $p$ value of $<0.00001$.

\section{CONCLUSIONS}

The incidence of post cross clamp ventricular fibrillation requiring electrical defibrillation is significantly lower with del Nido cardioplegia compared with conventional blood cardioplegia in isolated coronary artery bypass surgery. This shows that del Nido cardioplegia is preferred for avoiding post cross clamp ventricular fibrillation and thereby preventing myocardial injury.

\section{KEY WORDS}

del Nido Cardioplegia, Ventricular Fibrillation, Post Cross Clamp
Corresponding Author: Dr. Anandakuttan Sreenivasan, Department of Cardiovascular and Thoracic Surgery, Government T D Medical College, Alappuzha, Kerala, India, E-mail:dranand75@gmail.com

DOI: $10.14260 /$ jemds/2020/797

How to Cite This Article:

Thyagarajan BK, Sreenivasan A, Jayakumar $R$, et al. Incidence of post cross clamp ventricular fibrillation in isolated coronary artery bypass surgery using del nido cardioplegia and conventional blood cardioplegia. J Evolution Med Dent Sci 2020;9(48):3628-3632, DOI: $10.14260 /$ jemds/2020/797

Submission 20-08-2020,

Peer Review 14-10-2020,

Acceptance 20-10-2020,

Published 30-11-2020.

Copyright (C) 2020 Biju Kambil Thyagarajan et al. This is an open access article distributed under Creative Commons Attribution License [Attribution 4.0 International (CC BY 4.0)] 


\section{BACKGROUND}

The greatest hurdle in the development of open-heart surgery was getting a motionless and blood less heart during surgery. With introduction of cardiopulmonary bypass by John Gibbon in 1953, we were able to transfer the function of heart and lung during cardiac surgery. Still getting a motionless heart during surgery with adequate myocardial protection without adverse effects on myocardial function in the post-operative period was big concern. The term 'cardioplegia' was first introduced by Lam in 1957. High potassium level is the key ingredient in all types of cardioplegia. Various types of high potassium containing solution with a variety of additives and varying concentration of potassium have been evaluated as methods for cardioplegia and myocardial protection. With the introduction of cardiopulmonary bypass and myocardial protection strategy using cardioplegia, the open-heart surgical procedures witnessed a dramatic reduction in morbidity and mortality. Even though the cardioplegia offered a motionless heart for the surgical procedure, it is associated with varying degrees of myocardial ischaemia and myocardial injury affecting post-operative myocardial function.

Today, various cardioplegia techniques are used for open heart surgery procedures. ${ }^{1}$ The cardioplegia solutions are classified as either extracellular or intracellular based on their ion composition and the cellular environment they reflect. Extracellular cardioplegia solutions include del Nido, St. Thomas, and Buckberg solutions, while Custodial HTK solution is an intracellular cardioplegia solution.

The conventional blood cardioplegia is prepared by mixing the crystalloid cardioplegia solution with blood in a 1:4 ratio and is administered every 20 minutes. The surgery has to be interrupted during the administration of repeated doses. The del Nido cardioplegia was developed by Pedro del Nido and his team at University of Pittsburgh for use in paediatric cardiac surgery in early 1990s. It is a blood and crystalloid formula and associated with prolonged myocardial ischemic time of more than 90 minutes. It contains various additives including magnesium and lidocaine, which inhibit intracellular accumulation of calcium and prevent ischemia-reperfusion injury after cardiac surgery. ${ }^{2,3}$ After expiration of its patent, the paediatric del Nido cardioplegia has been increasingly used in adult cardiac surgery. The unique promising feature of del Nido cardioplegia in adult cardiac surgery is the longer redosing intervals up to 90 minutes and the surgery can be done without interruption.

Ventricular fibrillation developing immediately after release of aortic cross clamp during coronary artery bypass surgery is a common energy spending phenomenon leading to rapid myocardial energy depletion in an already energy deficient myocardium due to aortic cross clamping and cardioplegia. Such ventricular fibrillation can lead to increased oxygen consumption, compromise coronary blood flow, subendocardial ischemia and can cause increased myocardial wall tension. All these will lead to further depletion of myocardial energy reserve. That is why it must be treated immediately with defibrillation. The DC (Direct-Current) cardioversion can cause myocardial injury. Dahl and associates demonstrated this by elevated levels of CPK-MB (Creatine Phospho-Kinase Myocardial Band). ${ }^{4}$ These two myocardial insults adversely affect the post-operative myocardial function and surgical outcome and hence, prevention of occurrence of ventricular fibrillation immediately after aortic cross clamp is more important than its treatment.

The aetiology of post de-clamping ventricular fibrillation is multifactorial. Major contributing factors are myocardial ischaemia and metabolic changes associated with aortic cross clamping. Other important factors include systemic temperature, serum potassium level and presence of membrane stabilising antiarrhythmic drugs such as lidocaine in the cardioplegia solution. Different drugs with membrane stabilising and antiarrhythmic action have been added to cardioplegia solution in an attempt to prevent occurrence of ventricular fibrillation after release of aortic cross clamp.

We wanted to compare the incidence of post cross clamp ventricular fibrillation requiring DC cardioversion in isolated coronary artery bypass using del Nido cardioplegia and conventional blood cardioplegia solutions.

\section{METHODS}

\section{Study Design}

This retrospective study was conducted in a single tertiary care academic centre, by analysing the data of patients who underwent isolated coronary artery revascularisation surgery from January 2011 to October 2018. The study design and protocol were submitted for approval before Institutional Research Committee and Institutional Ethics Committee and was approved. The study population was organised into two groups based on the type of cardioplegia strategy during surgery: Group I intermittent conventional blood cardioplegia used in patients from January 2011 to September 2016, Group II del Nido cardioplegia used in patients from October 2016 to October 2018. We selected first 100 patients in each group and data was collected from the hospital records.

The exclusion criteria were previous dysrhythmias, emergency surgery, off pump coronary artery bypass surgery, re-do surgeries, non-CABG (Coronary Artery Bypass Surgery) cardiac surgeries, combined surgeries and surgeries using cardioplegia other than blood cardioplegia and del Nido cardioplegia.

\section{Cardioplegia}

The conventional cardioplegia is prepared by adding $30 \mathrm{~mL}$ of potassium chloride ( $2 \mathrm{mEq} / \mathrm{mL}$ ), $62.5 \mathrm{~mL}$ of $20 \%$ mannitol and $30 \mathrm{~mL}$ of $8.4 \%$ sodium bicarbonate to $1000 \mathrm{~mL}$ of $5 \%$ dextrose and diluting it with the subjects heparinised oxygenated blood from the cardiopulmonary bypass circuit in the ratio 1:4 (crystalloid: blood), cooled to $4^{0} \mathrm{C}$ and administered antegrade into the root of aorta. The first dose is $20 \mathrm{~mL} / \mathrm{kg}$ of body weight and repeated every $25-30$ minutes with a dose of $10 \mathrm{~mL} / \mathrm{kg}$ of body weight.

The del Nido cardioplegia is prepared by adding $13 \mathrm{~mL}$ of potassium chloride ( $2 \mathrm{mEq} / \mathrm{mL}$ ), $16.3 \mathrm{~mL}$ of $20 \%$ mannitol, $13 \mathrm{~mL}$ of $8.4 \%$ sodium bicarbonate, $4 \mathrm{~mL}$ of $50 \%$ magnesium sulphate, and $13 \mathrm{~mL}$ of $1 \%$ lidocaine to $1000 \mathrm{~mL}$ of PlasmaLyte A and diluting it with subjects heparinised oxygenated blood from the extracorporeal circuit in the ratio $4: 1$ (crystalloid: blood), cooled to $4^{\circ} \mathrm{C}$ and administered antegrade into the aortic root. Initial dosing was $1000 \mathrm{~mL}$ and re-dosing is considered when aortic occlusion time exceeds 90 minutes. $^{3}$ 


\section{Post-Cross Clamp Ventricular Fibrillation}

After putting standard invasive monitoring lines, the patient is administered general anaesthesia. The surgery is performed through a standard midline sternotomy. Left internal mammary artery and long saphenous veins are harvested. After heparinisation with 300 units / $\mathrm{kg}$ of heparin to maintain ACT (Activated Clotting Time) more than 400, cardiopulmonary bypass is instituted in the standard fashion with aortic and atriocaval cannulation. After attaining full flows, aortic occlusion clamp is applied and the cardioplegic solution is delivered antegrade in to the root of aorta over a period of 3 to 5 minutes. All patients were cooled to $30^{\circ} \mathrm{C}$. Ice slush is used for topical cooling of myocardium. During cardiopulmonary bypass the mean blood pressure should be maintained between 50 and $70 \mathrm{~mm}$ of $\mathrm{Hg}$. After completing distal anastomosis, patient is rewarmed to $36^{\circ} \mathrm{C}$ and aortic cross clamp is released. If ventricular fibrillation develops after release of aortic cross clamp it is electrically cardioverted with $10-20$ Joules of internal shock. The proximal ends of venous grafts are anastomosed to ascending aorta after applying partially occluding aortic clamp. Once the patient is rewarmed to normal temperature, the patient is gradually weaned from cardiopulmonary bypass after assessing myocardial contractility, arterial blood gases and electrolytes. Inotropic supports are used to maintain optimal cardiac output. Once haemodynamic stability is achieved, action of heparin is reversed using protamine. After achieving haemostasis, the sternal wound is closed after putting drains and epicardial pacing wire and the patient is shifted to cardiac surgery ICU.

\section{Data Collection}

Specific pre-operative, intraoperative and post-operative variables were collected using structured proforma. Preoperative patient parameters collected are detailed in Table 1.

\section{Statistical Analysis}

The patients' data was collected and entered in Microsoft Excel. The statistical analysis was done using SPSS version 10. Two samples t-test and chi-square test are used for analysis of variables to assess level of significance. All $p$ values $\leq 0.05$ were considered significant.

\section{RESULTS}

This retrospective study analysed the data of total of 200 patients who underwent elective isolated coronary artery bypass graft surgery: 100 in the Conventional Blood Cardioplegia (CBCP) group and 100 in the del Nido Cardioplegia (DNCP) group. The preoperative patient demographics in two groups are presented in Table 1 . The preoperative parameters were similar in two groups and doesn't show any statistically significant difference. Four patients in the del Nido cardioplegia group and 24 patients in the conventional blood cardioplegia group developed ventricular fibrillation after release of aortic occlusion clamp and required electrical cardioversion to revert the ventricular fibrillation to sinus rhythm. The incidence of post-cross clamp ventricular fibrillation requiring defibrillation in two groups were analysed using two sample $\mathrm{T}$ test and is significantly low in the DNCP group with a p value of $<0.00001$ (Table 2)

\begin{tabular}{|ccccc|}
\hline Sl. No & Parameter & CBCP & DNCP & P Value \\
1 & AGE & $56.38 \pm 7.44$ & $57.96 \pm 7.76$ & 0.2398 \\
2 & SEX & M: F $22: 3$ & $21: 4$ & 0.4151 \\
3 & BSA & $1.71 \pm 0.15$ & $1.70 \pm 0.15$ & 0.7247 \\
4 & LMCA Disease & 41 & 43 & 0.7741 \\
5 & Number of Coronary Arteries & $2.81 \pm 0.40$ & $2.67 \pm 0.58$ & 0.0863 \\
6 & Involved & 54 & 52 & 0.7772 \\
7 & DM & 56 & 59 & 0.6679 \\
8 & HTN & 35 & 42 & 0.3092 \\
9 & DLP & $62.27 \pm 11.17$ & $61.50 \pm 9.83$ & 0.6710 \\
\hline \multicolumn{4}{c}{} \\
\hline
\end{tabular}

\begin{tabular}{|c|c|c|c|}
\hline Parameter & CBCP & DNCP & P Value \\
\hline Ventricular fibrillation & 24 & 4 & $<0.00001$ \\
\hline \multicolumn{4}{|c|}{ Table 2. Ventricular Fibrillation after Release of Aortic Cross Clam } \\
\hline
\end{tabular}

del Nido cardioplegia is an extracellular cardioplegia solution mixed with patient blood from the CPB in a 4:1 ratio, cooled to $4^{\circ} \mathrm{C}$ and administered to the root of aorta after aortic cross clamping. Each ingredient in del Nio cardioplegia has an important role in myocardial protection. The Plasma-Lyte A (Baxter International Inc.) forms the crystalloid base of DNCP. It is a balanced extracellular solution with a $\mathrm{pH}$ of 7.4 and osmolarity of $294 \mathrm{mOsmol} / \mathrm{L}$. It has an electrolyte composition similar to extracellular fluid. Its composition is given in Table 4. The major advantage of the solution is that it is calcium free and the final concentration of calcium after mixing 4 part with one-part blood is only trace. Traces of calcium in the cardioplegia has shown to minimise myocardial injury during cardioplegic arrest compared to normocalcaemic or acalcaemic cardioplegia.5, 6

The high potassium concentration is responsible for the rapid depolarisation arrest and prompt cessation of myocardial contractility, there by preservation of myocardial energy supplies. ${ }^{7}$ The high potassium concentration in the cardioplegia decreases the resting membrane potential of myocardial cells. When cardioplegia is administered after aortic cross clamping, it will displace the normokalaemic blood surrounding cardiac myocytes, the membrane voltage become less negative from its normal - $90 \mathrm{mV}$ and the cell depolarises more rapidly leading to myocardial contraction. Following this the intracellular calcium will be sequestrated by sarcoplasmic reticulum via ATP dependent $\mathrm{Ca}^{2+}$ pumps and the myocardium relaxes. Further repolarisation of cardiac myocytes is prevented by the hyperkalaemic cardioplegic solution surrounding the myocytes. Very high levels of potassium in the cardioplegia solution will bring the membrane potential to $-50 \mathrm{mV}$ leading to inactivation of sodium channel resulting in diastolic arrest of heart.

The initial rapid depolarisation (phase 0) of myocardial action potential is due to opening of voltage gated $\mathrm{Na}^{+}$ channels. Lidocaine is a Class Ib antiarrhythmic drug that directly blocks $\mathrm{Na}$ channels in the phase 0 of action potential of cardiac myocyte. Lidocaine has only a modest depressant action on the rate of rise of action potential in Phase 0 . But presence of hyperkalaemia increases the ability of lidocaine to block fast $\mathrm{Na}^{+}$channels. Lidocaine increases cardiac muscle refractory period and counteracts the negative effects of hyperkalaemic depolarised arrest by polarising the myocardial cell and by reducing intracellular influx of sodium and calcium. ${ }^{8}$ It has a relatively long half-life which is 
increased in the presence of absent coronary circulation after aortic cross clamping providing prolonged cardiac arrest. Magnesium is a natural calcium channel blocker. Influx of extracellular $\mathrm{Ca}^{2+}$ triggered by activation of dihydropyridine channels in the $\mathrm{T}$ system triggers released of $\mathrm{Ca}^{2+}$ stored in the sarcoplasmic reticulum and during relaxation it is pumped back into the sarcoplasmic reticulum. Accumulation of $\mathrm{Ca}^{2+}$ in the myocardium can cause interruption of relaxation leading to myocardial stiffness and poor myocardial recovery. Magnesium prevents accumulation of calcium within the cells ${ }^{8,9}$ and improves myocardial recovery. Mannitol prevents myocardial injury during cardioplegia and reperfusion. ${ }^{10}$ The oxygen free radical produced during the cardioplegic arrest and reperfusion includes superoxide anion, hydrogen peroxide and hydroxyl. These are normally neutralised enzymatically within the cells. But this is inhibited during cardiac arrest. The hyperosmolar mannitol in the del Nido cardioplegia will scavenge the free radicals and reduce myocardial cellular oedema. The blood added to the del Nido cardioplegia promotes aerobic metabolism, helps buffering action to promote anaerobic glycolysis by buffering excess hydrogen ions, improves coronary artery perfusion during delivery, preserves myocardial metabolism and reduces myocardial injury during reperfusion. Sodium bicarbonate helps to scavenge excess hydrogen ion produced during anaerobic glycolysis and helps to maintain $\mathrm{pH}$. This buffering action will promote anaerobic glycolysis during cardiac arrest period as the elevated hydrogen ion has been shown to inhibit anaerobic glycolysis and thereby ATP production. More over the high concentration of carbonic anhydrase present in the red blood cells along with sodium bicarbonate helps to buffer the hydrogen ions produced during cardioplegic arrest.

\begin{tabular}{|ccc|}
\hline Parameter & CBCP & DNCP \\
\hline Carrier & $5 \%$ Dextrose in Water $1000 \mathrm{~mL}$ & Plasma-Lyte A $1000 \mathrm{~mL}$ \\
Blood: $\mathrm{CP}$ ratio & $4: 1$ & $1: 4$ \\
$\mathrm{KCl}$ & $60 \mathrm{mmol}$ & $26 \mathrm{mmol}$ \\
$\mathrm{NaHCO}_{3}$ & $30 \mathrm{mmol}$ & $13 \mathrm{mmol}$ \\
Mannitol $_{\text {Lidocaine }}$ & $12.5 \mathrm{gm}(68.62 \mathrm{mmol})$ & $3.26 \mathrm{gm}(17.90 \mathrm{mmol})$ \\
\hline $\mathrm{MgSO}_{4}$ & 0 & $130 \mathrm{mg}(0.55 \mathrm{mmol})$ \\
\hline \multicolumn{2}{r}{ Table 3. Composition of Cardioplegia Solutions } \\
\hline
\end{tabular}

\begin{tabular}{|cc|}
\hline Composition & Plasma-Lyte A \\
Ph & 7.4 \\
Sodium (mEq / L) & 140 \\
Potassium (mEq / L) & 5 \\
Calcium (mEq / L) & 0 \\
Magnesium (mEq / L) & 3 \\
Chloride (mEq / L) & 98 \\
Lactate (mEq / L) & 0 \\
\hline Gluconate (mEq / L) & 23 \\
Acetate (mEq / L) & 27 \\
Osmolarity (mosmol / L) & 294 \\
\hline Table 4. Composition of Plasma-Lyte A \\
\hline
\end{tabular}

\begin{tabular}{|ccc|}
\hline Final Composition & CBCP & DNCP \\
$\mathrm{K} \mathrm{(mmol} \mathrm{/} \mathrm{L)}$ & 13.61 & 24.0 \\
$\mathrm{Mg}(\mathrm{mmol} / \mathrm{L})$ & 0.66 & 6.30 \\
$\mathrm{Ca}(\mathrm{mmol} / \mathrm{L})$ & 1.8 & 0.45 \\
Lidocaine (mmol / L) & 0 & 0,42 \\
\hline Table 5. Estimated Final Composition of Cardioplegia \\
\hline
\end{tabular}

Lidocaine and magnesium will prevent intracellular accumulation of calcium ion during myocardial arrest and there by avert myocardial injury and ventricular fibrillation associated with reperfusion. In the present study the incidence of post cross clamp ventricular fibrillation is significantly low with del Nido cardioplegia technique compared to conventional blood cardioplegia which uses a lidocaine and magnesium free cardioplegia solution.

\section{DISCUSSION}

Cardioplegia induced cardiac arrest is a non-physiological state and the heart is extremely vulnerable to ventricular fibrillation immediately after declamping of aorta. The mechanism of ventricular fibrillation immediately after aortic declamping is not precisely understood. Many factors contribute to this such as, electrolyte imbalances, acid disturbances, conduction abnormalities and most importantly inadequate myocardial protection. ${ }^{11,12}$ The ideal method for myocardial protection during aortic cross clamping is an area of ongoing research. The type of cardioplegic solution used have a role in preventing or minimising myocardial injury during this time. The degree of myocardial ischemia during open heart surgery and there by the type of cardioplegia used greatly affects development of ventricular fibrillation after aortic declamping and the ventricular function after surgery. ${ }^{13}$

The available literature supports our study findings. Kavala AA, et al compared the del Nido cardioplegia with blood cardioplegia in coronary artery bypass surgery combined with mitral valve replacement and demonstrated significantly lower incidence of need for intraoperative defibrillation. ${ }^{14}$ Kumar et al compared del Nido cardioplegia with St. Thomas's cardioplegia for myocardial protection in adult open heart surgery and reported a significantly lower requirement of intraoperative cardioversion with del Nido cardioplegia. ${ }^{15}$

\section{CONCLUSIONS}

Our study has shown that in patients who underwent isolated coronary artery bypass grafting surgery using del Nido cardioplegia, the incidence of ventricular fibrillation requiring electrical cardioversion after removal aortic cross clamp was significantly lower than with conventional blood cardioplegia.

Data sharing statement provided by the authors is available with the full text of this article at jemds.com.

Financial or other competing interests: None.

Disclosure forms provided by the authors are available with the full text of this article at jemds.com.

We would like to thank all the colleagues who helped us in collection and analysis of the data.

\section{REFERENCES}

[1] Melrose DG, Dreger B, Bentall HH, et al. Elective cardiac arrest. Lancet 1955;269(6879):21-2.

[2] Matte GS, del Nido PJ. History and use of del Nido cardioplegia solution at Boston Children's Hospital. J Extra Corpor Technol 2012;44(3):98-103.

[3] Yerebakan H, Sorabella RA, Najjar M, et al. del Nido Cardioplegia can be safely administered in high - risk coronary artery bypass grafting surgery after acute 
myocardial infarction: a propensity matched comparison. J Cardiothorac Surg 2014;9:141.

[4] Dahl CF, Ewy GA, Warner ED, et al. Myocardial necrosis from direct current countershock. Effect of paddle electrode size and time interval between discharges. Circulation 1974;50(5):956-61.

[5] O'Blenes SB, Friesen CH, Ali A, et al. Protecting the aged heart during cardiac surgery: the potential benefits of del Nido cardioplegia. J Thorac Cardiovasc Surg 2011;141(3):762-70.

[6] Jennings RB, Reimer KA. Lethal myocardial ischemic injury. Am J Pathol 1981;102(2):241-55.

[7] Pragliola C, Hassan E, Al Hossan A, et al. del Nido cardioplegia: from an infant conceive to an adult life - a brief review of the current evidence in adult patients. Vessel Plus 2019;3:22.

[8] Larach DR, Solina AR. Cardiovascular drugs. In: Hensley FA, Martin DE, eds. A practical approach to cardiac anesthesia. $2^{\text {nd }}$ edn. Boston: Little, Brown and Company 1995:32-95.

[9] Iseri LT, French JH. Magnesium: nature's physiologic calcium blocker. Am Heart J 1984;108(1):188-93.
[10] Powell WJ, Di Bona DR, Flores J. The protective effect of hyperosmotic mannitol in myocardial ischemia and necrosis. Circulation 1976;54(4):603-15.

[11] Ristagno G, Wang T, Tang W, et al. High - energy defibrillation impairs myocyte contractility and intracellular calcium dynamics. Crit Care Med 2008;36(11 Suppl):S422-7.

[12] Jynge P, Sellevold OF. Cardioplegia: mechanisms of protection revisited. Ann Chi Gynaecol 1987;76(1):22-9.

[13] Kim JS, Jeong JH, Moon SJ, et al. Sufficient myocardial protection of del Nido cardioplegia regardless of ventricular mass and myocardial ischemic time in adult cardiac surgical patients. J Thorac Dis 2016;8(8):2004-10.

[14] Kavala AA, Turkyilmaz S. del Nido cardioplegia for CABG combined with mitral valve surgery. Braz J Cardiovasc Surg 2018;33(5):496-504.

[15] Kumar A, Pandey AR, Chandra S, et al. Comparison of del Nido's cardioplegia with St. Thomas's cardioplegia for myocardial protection in adult open-heart surgery. Heart India 2019;7(4):145-9. 